\title{
FULL FACTORS AND CO-AMENABLE INCLUSIONS
}

\author{
JON BANNON, AMINE MARRAKCHI, AND NARUTAKA OZAWA
}

\begin{abstract}
We show that if $M$ is a full factor and $N \subset M$ is a coamenable subfactor with expectation, then $N$ is also full. This answers a question of Popa from 1986. We also generalize a theorem of Tomatsu by showing that if $M$ is a full factor and $\sigma: G \curvearrowright M$ is an outer action of a compact group $G$, then $\sigma$ is automatically minimal and $M^{G}$ is a full factor which has w-spectral gap in $M$. Finally, in the appendix, we give a proof of the fact that several natural notions of co-amenability for an inclusion $N \subset M$ of von Neumann algebras are equivalent, thus closing the cycle of implications given in Anantharaman-Delaroche's paper in 1995.
\end{abstract}

\section{INTRODUCTION}

An inclusion of two von Neumann algebras $N \subset M$ is called co-amenable if the inclusion of the commutants $M^{\prime} \subset N^{\prime}$ admits a conditional expectation $\Phi: N^{\prime} \rightarrow M^{\prime}$. In particular, $M$ is amenable if and only if the inclusion $\mathbf{C} \subset M$ is co-amenable. This notion originally appeared in Definition 3.2.1 of [Po86], and was there called relative amenability. In MoPo03, the relation between this concept and the notion of co-amenability for groups was clarified. Indeed, an inclusion of groups $H \subset G$ co-amenable if and only if there exists a $G$-invariant mean on $\ell^{\infty}(G / H)$, and it holds that the inclusion of group von Neumann algebras $L(H) \subset L(G)$ is co-amenable if and only if the inclusion $H \subset G$ is co-amenable. Other examples of co-amenable inclusion are given by finite index inclusions, inclusions of the form $N \subset N \rtimes G$ with $G$ amenable or inclusions of the form $M^{G} \subset M$ for a minimal compact group action $G \curvearrowright M$.

Following Co74, we say that a separable factor $M$ is full if the group of all its inner automorphisms $\operatorname{Inn}(M)$ is closed in $\operatorname{Aut}(M)$. In the type $\mathrm{II}_{1}$ case, the factor $M$ is full if and only if it does not have the property Gamma of Murray and von Neumann MvN43, i.e. if and only if every central sequence of $M$ is trivial, or equivalently, if and only if $M^{\prime} \cap M^{\omega}=\mathbf{C}$ where $M^{\omega}$ is the ultrapower of $M$ with respect to a free ultrafilter on $\mathbf{N}$. Fullness is also closely related to a group-theoretic notion. Recall that a group $G$ is inner amenable when there exists a non-trivial conjugacy invariant mean

2010 Mathematics Subject Classification. 46L10, 46L36, 46L40, 46L55.

Key words and phrases. von Neumann Algebras; Full factor; co-amenable inclusion; compact group; minimal action.

JB is partially supported by a Lancaster University (STEM) Fulbright Scholar Award.

AM is a JSPS International Research Fellow (PE18760).

NO is partially supported by JSPS KAKENHI $17 \mathrm{~K} 05277$ and $15 \mathrm{H} 05739$. 
on $\ell^{\infty}(G)$. By [Ef73, if $G$ is non-inner amenable then $L(G)$ is a full factor. A subtle counter-example due to Vaes Va09 shows that the converse implication is however not true.

It is not difficult to see that if $H \subset G$ is a co-amenable group inclusion and if $H$ is inner amenable, then $G$ must also be inner amenable. In Po86, Problem 3.3.2], Popa asked the following analogous question in the context of von Neumann algebras:

Question ([Po86]). If $M$ is a full $\mathrm{II}_{1}$ factor and $N \subset M$ is a co-amenable subfactor, is it true that $N$ is also full?

This question was partially motivated by Proposition 1.4.1 of [Po83], which affirmatively answers the above question when $M$ is the crossed product of $N$ by an action of $\mathbf{Z}$. A few years later (cf. Proposition 1.11 of [PiPo86]), he and Pimsner proved that when $N \subset M$ is a finite index inclusion of $\mathrm{II}_{1}$ factors then $N$ is full if and only if $M$ is full. The case where the co-amenable inclusion $N \subset M$ is regular and irreducible was confirmed by Bédos [Be90, and independently by Bisch [Bi90]. Such subfactors arise as cocycle crossed products of free cocycle actions of amenable groups on $\mathrm{II}_{1}$ factors (cf. Ch79]), and both Bédos's and Bisch's proofs rely heavily on this. We note that the specialized question for crossed products by amenable groups also follows from the recent work of the second author, which completely characterizes fullness of crossed product factors $N \rtimes G$ with $N$ a factor of arbitrary type and $G$ discrete amenable [Ma18. In that case, not only $N$ is full, but it also has spectral gap in $N \rtimes G$.

We now state our main theorem. We assume that our inclusion $N \subset$ $M$ is with expectation, i.e. that there exists a faithful normal conditional expectation $\mathrm{E}_{N}: M \rightarrow N$. This is automatic if $M$ is tracial, but it is both a necessary and a natural assumption in the non-tracial setting.

Theorem A. Let $M$ be a separable full factor and $N \subset M$ a co-amenable subalgebra with expectation. Then there exists a non-zero projection $p \in$ $N^{\prime} \cap M$ such that $p\left(N^{\prime} \cap M^{\omega}\right) p=\mathbf{C} p$ for all $\omega \in \beta \mathbf{N}$.

In particular, we obtain a complete solution to Popa's question, but for factors of arbitrary type and with the additional property that $N$ has $w$ spectral gap in $M$ in the irreducible case.

Corollary B. Let $M$ be a separable full factor and let $N \subset M$ be a coamenable subfactor with expectation. Then $N$ is full. Moreover, if $N^{\prime} \cap M=$ $\mathbf{C}$, then we have $N^{\prime} \cap M^{\omega}=\mathbf{C}$ for all $\omega \in \beta \mathbf{N}$.

We note that our proof is completely different from the case of crossed products by amenable groups. Indeed, in that specific case, Bisch proves that if $N$ is not full then $M^{\prime} \cap N^{\omega} \neq \mathbf{C}$. But he subsequently (cf. [Bi94]) exhibits a finite index inclusion $N \subset M$ of hyperfinite $\mathrm{II}_{1}$ factors such that $M^{\prime} \cap N^{\omega}=\mathrm{C}$, hence showing that one cannot hope to solve the general problem by proving that $M^{\prime} \cap N^{\omega} \neq \mathbf{C}$ for all co-amenable inclusions of $\mathrm{II}_{1}$ factors $N \subset M$ where $N$ is not full. Instead, the proof of Theorem $\mathrm{A}$ essentially encodes a reduction of the problem to the finite index case. We also note that in the case of crossed products of $\mathrm{II}_{1}$ factors by amenable groups, one can actually show that the subfactor $N \subset M$ has spectral gap 
thanks to Jo81 and Ma18. In Remark 3.6, we observe that this is no longer true for arbitrary co-amenable subfactors: in general the conclusion $N^{\prime} \cap M^{\omega}=\mathbf{C}$ of Corollary $\mathrm{B}$ cannot be improved to true spectral gap.

In our second main result, we give an application of Theorem $\mathrm{A}$ to actions of compact groups on full factors. Very recently, Tomatsu proved that if $\sigma: G \curvearrowright M$ is a minimal action of a compact group $G$ on a full factor $M$, then $M^{G}$ and $M \rtimes G$ are full factors [To18, Theorem 4.8]. Recall that an action is minimal if it is faithful and $\left(M^{G}\right)^{\prime} \cap M=\mathbf{C}$. A minimal action is necessarily outer meaning that $\sigma_{g} \notin \operatorname{Inn}(M)$ for all $g \neq 1$. But the converse is far from being true in general. Nevertheless, thanks to Theorem A, we can show that outerness automatically implies minimality for actions of compact groups on full factors. This is the content of our second main theorem. We also strengthen Tomatsu's result by showing that $M^{G}$ has w-spectral gap in $M$.

Theorem C. Let $\sigma: G \curvearrowright M$ be an outer action of a compact group $G$ on a separable factor $M$. Assume that $M$ is full. Then $\sigma$ is automatically minimal and $M^{G}$ and $M \rtimes G$ are full factors. Moreover, we have $\left(M^{G}\right)^{\prime} \cap M^{\omega}=\mathbf{C}$ and $M^{\prime} \cap(M \rtimes G)^{\omega}=\mathbf{C}$ for all $\omega \in \beta \mathbf{N}$.

To obtain this theorem, we first prove the following dichotomy: for any outer action $\sigma: G \curvearrowright M$ of a compact group $G$ on an arbitrary factor $M$, either $\left(M^{G}\right)^{\prime} \cap M=\mathbf{C}$ or $\left(M^{G}\right)^{\prime} \cap M$ is diffuse. Then we apply Theorem $\mathrm{A}$,

Acknowledgments. The first author thanks Jan Cameron for sharing his ideas on Property $\Gamma$ for an earlier approach to the problem, and is grateful for the hospitality of the Lancaster University Department of Mathematics and Statistics. The second author is grateful to Yuki Arano for a thoughtprovoking discussion regarding Theorem 4.1. We are also grateful to Adrian Ioana for providing us with the reference [HK05] used in Remark 3.6.

\section{Preliminaries}

2.1. Ultrapowers. Given a free (i.e. nonprincipal) ultrafilter $\omega \in \beta \mathbf{N} \backslash \mathbf{N}$ and $\sigma$-finite von Neumann algebra $M$ one defines

$$
\mathcal{I}_{\omega}(M)=\left\{\left(x_{n}\right)_{n} \in \ell^{\infty}(\mathbf{N}, M) \mid \lim _{n \rightarrow \omega} x_{n}=0 * \text {-strongly }\right\}
$$

and its multiplier algebra

$$
\mathfrak{M}^{\omega}(M)=\left\{\left(x_{n}\right)_{n} \in \ell^{\infty}(\mathbf{N}, M) \mid\left(x_{n}\right)_{n} \mathcal{I}_{\omega}(M)+\mathcal{I}_{\omega}(M)\left(x_{n}\right)_{n} \subset \mathcal{I}_{\omega}(M)\right\} .
$$

The latter is a $C^{*}$-algebra inside of which the former is a norm-closed twosided ideal. The quotient $C^{*}$-algebra $M^{\omega}:=\mathfrak{M}^{\omega}(M) / \mathcal{I}_{\omega}(M)$ is actually a von Neumann algebra and is called the Ocneanu ultrapower of $M$ associated to $\omega$ Oc85. The von Neumann algebra $M$ naturally embeds into $M^{\omega}$ as $x \mapsto\left(x_{n}\right)_{n}$ with $x_{n}=x$ for all $n \in \mathbf{N}$.

The Ocneanu ultrapower generalizes the tracial ultrapower associated to a finite von Neumann algebra $M$ with trace $\tau$. Indeed, in that specific case we have $\mathcal{I}_{\omega}(M)=\left\{\left(x_{n}\right)_{n} \in \ell^{\infty}(\mathbf{N}, M) \mid \lim _{n \rightarrow \omega}\left\|x_{n}\right\|_{2}=0\right\}$ and $\mathfrak{M}^{\omega}(M)=$ $\ell^{\infty}(\mathbf{N}, M)$, and the Ocneanu ultrapower admits the faithful normal trace $\tau_{\omega}\left(\left(x_{n}\right)_{n}+\mathcal{I}_{\omega}(M)\right)=\lim _{n \rightarrow \omega} \tau\left(x_{n}\right)$. 
2.2. Standard form and basic construction. For any von Neumann algebra $M$, we denote by $\left(M, \mathrm{~L}^{2}(M), J, \mathrm{~L}^{2}(M)^{+}\right)$its standard form [Ha73. Recall that $\mathrm{L}^{2}(M)$ is naturally endowed with the structure of a $M-M$ bimodule: we will simply write $x \xi y=x J y^{*} J \xi$ for all $x, y \in M$ and all $\xi \in \mathrm{L}^{2}(M)$. We will denote by $\lambda: M \rightarrow \mathbf{B}\left(\mathrm{L}^{2}(M)\right)$ and $\rho: M^{\mathrm{op}} \rightarrow \mathbf{B}\left(\mathrm{L}^{2}(M)\right)$ the associated left and right regular representation of $M$. We have $\lambda(M)=$ $\rho\left(M^{\mathrm{op}}\right)^{\prime}$. We will use the notation $C_{\lambda \cdot \rho}^{*}(M)$ for the $C^{*}$-algebra generated by $\lambda(M) \rho\left(M^{\mathrm{op}}\right)$.

If $N \subset M$ is a subalgebra, the so-called basic construction associated to it is the von Neumann algebra $\rho\left(N^{\mathrm{op}}\right)^{\prime}$, which will be denoted $\langle M, N\rangle$. Since $\lambda(M) \subset \rho\left(N^{\mathrm{op}}\right)^{\prime}$, we will view $M$ as a subalgebra of $\langle M, N\rangle$. When $N \subset M$ admits a faithful normal conditional expectation $\mathrm{E}_{N}: M \rightarrow N$, there is associated to it a canonical $N$-N-bimodular isometry $V: \mathrm{L}^{2}(N) \rightarrow \mathrm{L}^{2}(M)$. Its range projection $e_{N}=V V^{*} \in \mathbf{B}\left(\mathrm{L}^{2}(M)\right)$ is the Jones projection of $\mathrm{E}_{N}$. In that case, it holds that $e_{N} x e_{N}=\mathrm{E}_{N}(x) e_{N}$ for all $x \in M$ and $M e_{N} M$ is a dense $*$-subalgebra of $\langle M, N\rangle$. Moreover, we can identify $\mathrm{L}^{2}(\langle M, N\rangle)$ with the space $\mathrm{L}^{2}\left(\mathrm{E}_{N}\right)$ associated to the completely positive map $\mathrm{E}_{N}: M \rightarrow M$ via the Stinespring construction, i.e. as the Hilbert space separation and completion of $M \odot \mathrm{L}^{2}(M)$ under the semi-inner product defined on simple tensors by $\left\langle x_{1} \otimes \eta_{1}, x_{2} \otimes \eta_{2}\right\rangle_{\mathrm{E}_{N}}:=\left\langle\mathrm{E}_{N}\left(x_{2}^{*} x_{1}\right) \eta_{1}, \eta_{2}\right\rangle_{\mathrm{L}^{2}(M)}$ with the natural bimodule action given by $a(x \otimes \eta) b:=a x \otimes \eta b$ for $x_{1}, x_{2}, a, b, x \in M$ and $\eta_{1}, \eta_{2} \in \mathrm{L}^{2}(M)$.

2.3. Correspondences. Let $M$ and $N$ be von Neumann algebras. The opposite von Neumann algebra $N^{\mathrm{op}}=\left\{n^{\mathrm{op}}: n \in N\right\}$ is identical to $N$ as a Banach space, but with product $n_{1}^{\mathrm{op}} n_{2}^{\mathrm{op}}=\left(n_{2} n_{1}\right)^{\mathrm{op}}$ and involution $\left(n^{\mathrm{op}}\right)^{*}=\left(n^{*}\right)^{\mathrm{op}}$. An $M$ - $N$-correspondence is a $*$-representation $\pi_{\mathcal{H}}: M \odot$ $N^{\mathrm{op}} \rightarrow \mathbf{B}(\mathcal{H})$ that is normal in each variable, or equivalently a binormal $M$ - $N$-bimodule structure on $\mathcal{H}$.

We will say that an $M$-N-correspondence $\mathcal{H}$ is contained in another $\mathcal{K}$, written abusively $\mathcal{H} \subset \mathcal{K}$, if there exists an $M$-N-bimodular isometry $V: \mathcal{H} \rightarrow \mathcal{K}$. We will say that $\mathcal{H}$ is weakly contained in $\mathcal{K}$, written $\mathcal{H} \prec \mathcal{K}$, if we have $\left\|\pi_{\mathcal{H}}(T)\right\| \leq\left\|\pi_{\mathcal{K}}(T)\right\|$ for all $T \in M \odot N^{\mathrm{op}}$. Said another way, we have $\mathcal{H} \prec \mathcal{K}$ if and only if the matrix coefficients of $\mathcal{H}$ can be locally modeled using those of $\mathcal{K}$ : for all $\xi \in \mathcal{H}$, finite subsets $E \subset M, F \subset N$ and $\varepsilon>0$ there exist $n \in \mathbb{N}$ and $\left\{\eta_{1}, \ldots \eta_{n}\right\} \subset \mathcal{K}$ such that

$$
\left|\langle x \xi y, \xi\rangle-\sum_{i=1}^{n}\left\langle x \eta_{i} y, \eta_{i}\right\rangle\right|<\varepsilon
$$

for all $x \in E$ and $y \in F$. If $\mathcal{H}$ has a vector $\xi$ which is cyclic, i.e. such that $M \xi N$ is dense in $\mathcal{H}$, then it is enough to check the above criterion for $\xi$.

Two $M$ - $N$-correspondences $\mathcal{H}$ and $\mathcal{K}$ are weakly equivalent, written $\mathcal{H} \sim$ $\mathcal{K}$, if $\mathcal{H} \prec \mathcal{K}$ and $\mathcal{K} \prec \mathcal{H}$. Equivalently, $\mathcal{H} \sim \mathcal{K}$ if and only if $\left\|\pi_{\mathcal{H}}(T)\right\|=$ $\left\|\pi_{\mathcal{K}}(T)\right\|$ for all $T \in M \odot N^{\text {op }}$.

2.4. Intertwining bimodules and finite index subfactors. We will use the following classical criterion from Popa's intertwining theory to reduce our problem to the case of finite index subfactors ([PiPo86] $)$. We are interested in (i) $\Rightarrow$ (iv), for which we give a direct proof for the reader's convenience. 
Lemma 2.1 ( $\mathrm{Po01}, \mathrm{Po} 03)$. Let $M$ be a $\mathrm{II}_{1}$ factor and $N \subset M$ a subalgebra . The following are equivalent:

(i) $\mathrm{L}^{2}(M) \subset \mathrm{L}^{2}(\langle M, N\rangle)$ as an $M$ - $M$-bimodule.

(ii) There exists a normal conditional expectation $\Phi:\langle M, N\rangle \rightarrow M$.

(iii) $M \prec_{M} N$ in the sense of Popa's intertwining theory.

(iv) There exists a non-zero projection $p \in N^{\prime} \cap M$ such that $N p \subset p M p$ is an irreducible subfactor with finite index.

Proof of (i) $\Rightarrow$ (iv). Assume (i) holds. The normal conditional expectation $\Phi$ in (ii) is obtained by compressing elements of $\langle M, N\rangle$ to the $M$ - $M$-submodule $\mathrm{L}^{2}(M) \subset \mathrm{L}^{2}(\langle M, N\rangle)$. Since $M e_{N} M$ is ultraweakly dense in $\langle M, N\rangle$, the element $a:=\Phi\left(e_{N}\right) \in N^{\prime} \cap M$ is nonzero. Let $q \in N^{\prime} \cap M$ denote the support projection of $a$. We claim that $q\left(N^{\prime} \cap M\right) q$ is completely atomic. Suppose for the sake of a contradiction that there is a nonzero projection $q_{0} \leq q$ in $N^{\prime} \cap M$ such that $q_{0}\left(N^{\prime} \cap M\right) q_{0}$ is diffuse. Then for any $\varepsilon>0$, there are projections $q_{1}, \ldots, q_{n}$ in $N^{\prime} \cap M$ such that $\tau_{M}\left(q_{i}\right) \leq \varepsilon$ and $\sum_{i} q_{i}=q_{0}$. Put $q_{i}^{\text {op }}:=J q_{i} J \in M^{\prime} \cap\langle M, N\rangle$ and observe that $\Phi\left(q_{i}^{\text {op }}\right) \in M^{\prime} \cap M=\mathbb{C} 1$ and $q_{i} e_{N}=q_{i}^{\mathrm{op}} e_{N}$. Hence

$$
q_{0} e_{N} q_{0}=\left(\sum_{i} q_{i} q_{i}^{\mathrm{op}}\right) e_{N}\left(\sum_{j} q_{j} q_{j}^{\mathrm{op}}\right) \leq \sum_{i} q_{i} q_{i}^{\mathrm{op}}
$$

and so

$$
(\tau \circ \Phi)\left(q_{0} e_{N} q_{0}\right) \leq(\tau \circ \Phi)\left(\sum_{i} q_{i} q_{i}^{\mathrm{op}}\right)=\sum_{i} \tau_{M}\left(q_{i}\right) \Phi\left(q_{i}^{\mathrm{op}}\right) \leq \varepsilon .
$$

Since $\varepsilon>0$ was arbitrary, one obtains $q_{0} a q_{0}=\Phi\left(q_{0} e_{N} q_{0}\right)=0$, a contradiction. Thus one can find a minimal projection $p$ in $q\left(N^{\prime} \cap M\right) q$. Then, $N p \subset p M p$ is an irreducible subfactor and its basic construction is *isomorphic to $p p^{\mathrm{op}}\langle M, N\rangle p p^{\mathrm{op}}$ via $\mathrm{L}^{2}(p M p) \cong p p^{\mathrm{op}} \mathrm{L}^{2}(M)$, where $p^{\mathrm{op}}:=$ $J p J \in M^{\prime} \cap\langle M, N\rangle$. Since $\Phi\left(p^{\mathrm{op}} e_{N}\right)=\Phi\left(p e_{N}\right)=p a \neq 0$, the element $\Phi\left(p^{\text {op }}\right) \in M^{\prime} \cap M=\mathbb{C} 1$ is nonzero and $\Phi\left(p^{\text {op }}\right)^{-1} p^{\text {op }} \Phi(\cdot)$ defines a normal conditional expectation from $p p^{\mathrm{op}}\langle M, N\rangle p p^{\mathrm{op}}$ onto $p p^{\mathrm{op}} M p p^{\mathrm{op}} \cong p M p$. This proves that $N p \subset p M p$ has finite index.

2.5. Crossed products by locally compact groups. Let $\sigma: G \curvearrowright M$ be a continuous action of a locally compact group $G$ on a von Neumann algebra $M$. Let $M \rtimes_{\sigma, \text { alg }} G$ be the algebraic crossed product.

Let $\alpha: M \rightarrow M \bar{\otimes} \mathrm{L}^{\infty}(G)=\mathrm{L}^{\infty}(G, M)$ be the normal $*$-homomorphism which sends $x \in M$ to $g \mapsto \sigma_{g}^{-1}(x)$. Then there is a unique injective $*$-homomorphism

$$
\pi: M \rtimes_{\sigma, \text { alg }} G \rightarrow M \bar{\otimes} \mathbf{B}\left(\mathrm{L}^{2}(G)\right)
$$

such that $\left.\pi\right|_{M}=\alpha$ and $\left.\pi\right|_{G}=1 \otimes \lambda$ for all $g \in G$, where $\lambda: G \rightarrow \mathbf{B}\left(\mathrm{L}^{2}(G)\right)$ is the left regular representation of $G$.

By definition, the crossed product von Neumann algebra $M \rtimes_{\sigma} G$ is the unique von Neumann algebra containing $M \rtimes_{\sigma \text {,alg }} G$ as a dense $*$-subalgebra and such that $\pi$ extends to a faithful normal $*$-homomorphism $\pi: M \rtimes_{\sigma} G \rightarrow$ $M \bar{\otimes} \mathbf{B}\left(\mathrm{L}^{2}(G)\right)$.

Recall that if $M$ is a type III factor with faithful, normal semifinite weight $\omega$ and associated modular automorphism group $\left(\sigma_{t}\right)_{t \in \mathbf{R}}$, then $M \cong\left(M \rtimes_{\sigma}\right.$ 
$\mathbf{R}) \rtimes_{\widehat{\sigma}} \mathbf{R}$, and $c(M):=M \rtimes_{\sigma} \mathbf{R}$ is a semifinite von Neumann algebra called the continuous core of $M$.

Beyond this, in this paper we will only need to know the following fundamental facts:

- $\pi\left(M \rtimes_{\sigma} G\right)$ is the fixed point subalgebra of $M \bar{\otimes} \mathbf{B}\left(\mathrm{L}^{2}(G)\right)$ for the diagonal action $\sigma \otimes \rho: G \curvearrowright M \bar{\otimes} \mathbf{B}\left(\mathrm{L}^{2}(G)\right)$ where $\rho: G \curvearrowright \mathbf{B}\left(\mathrm{L}^{2}(G)\right)$ is the action coming from the conjugation by the right regular representation of $G$.

- There is a (surjective) $*$-isomorphism from the basic construction $\langle M \rtimes G, M\rangle$ to $M \bar{\otimes} \mathbf{B}\left(\mathrm{L}^{2}(G)\right)$ which restricts to $\pi$ on $M \rtimes_{\sigma} G$.

- If $G$ is compact, there exists a surjective $*$-homomorphism from $M \rtimes G$ onto the basic construction $\left\langle M, M^{G}\right\rangle$ which restricts to the identity on $M$ [Pa77, Theorem 4.2].

\subsection{Co-amenable inclusions.}

Definition 2.2. Let $M \subset \mathbf{B}(H)$ be a von Neumann algebra. We say that a subalgebra $N \subset M$ is co-amenable in $M$ if there exists a (not necessarily normal) conditional expectation $\Phi: N^{\prime} \rightarrow M^{\prime}$.

This property does not depend on the choice of the spatial realization $M \subset \mathbf{B}(H)$. Indeed, any other spatial realization can be obtained from $H$ by amplification and reduction. We then observe that $\Phi$ extends to a conditional expectation $\Phi \bar{\otimes} \mathrm{id}: N^{\prime} \bar{\otimes} \mathbf{B}(K) \rightarrow M^{\prime} \bar{\otimes} \mathbf{B}(K)$ for any Hilbert space $K$, and restricts to a conditional expectation $\left.\Phi\right|_{p N^{\prime} p}: p N^{\prime} p \rightarrow p M^{\prime} p$ for any projection $p \in M^{\prime}$.

We see immediately from the definition that the following properties are satisfied:

- If $N \subset P$ and $P \subset M$ are co-amenable then $N \subset M$ is co-amenable.

- If we have $N \subset P \subset M$ and $N \subset M$ is co-amenable then $P \subset M$ is co-amenable (but $N \subset P$ need not be co-amenable).

- If $p \in N$ is any projection whose central support in $N$ is 1 , then $p N p \subset p M p$ is co-amenable if and only if $N \subset M$ is co-amenable (because the map $x \mapsto p x$ is an isomorphism from $N^{\prime}$ to $p N^{\prime}$ ).

- $N \subset M$ is co-amenable if and only if there exists a conditional expectation $\Phi:\langle M, N\rangle \rightarrow M$. Indeed, by definition, the inclusion $M \subset\langle M, N\rangle$ is anti-isomorphic to $M^{\prime} \subset N^{\prime}$ in the standard representation $M \subset \mathbf{B}\left(\mathrm{L}^{2}(M)\right)$.

Crossed products by locally compact groups produce natural examples of co-amenable inclusions. For the proofs, we refer to Proposition 2.6 and Proposition 3.4 in AD95. We point out that our definition of co-amenability corresponds to what is called relative injectivity in AD95.

Theorem 2.3. Let $M$ be a von Neumann algebra and $\sigma: G \curvearrowright M$ and action of a locally compact group. Then the inclusion $M \rtimes G \subset\langle M \rtimes G, M\rangle$ is co-amenable, i.e. there exists a conditional expectation $\Phi: M \rtimes G \rightarrow M$.

If $G$ is amenable, then the inclusion $M \subset M \rtimes_{\sigma} G$ is also co-amenable, i.e. there exists a conditional expectation $\Phi:\langle M \rtimes G, M\rangle \rightarrow M \rtimes G$.

Finally, we need the following bimodule characterization of co-amenability. The proof is given in Proposition 2.5 and Proposition 3.6 of AD95] in the 
semifinite case and this is enough for our purpose. In the appendix, we include a proof for arbitrary von Neumann algebras.

Theorem 2.4. Let $N \subset M$ be an inclusion of von Neumann algebras. Then $N \subset M$ is co-amenable if and only if $\mathrm{L}^{2}(M) \prec{ }_{M} \mathrm{~L}^{2}(\langle M, N\rangle)_{M}$.

2.7. Full Factors. A factor $M$ is full if every central net in $M$ is trivial, i.e. if for every bounded net $\left(x_{i}\right)_{i}$ in $M$ such that $\left\|\varphi\left(\cdot x_{i}\right)-\varphi\left(x_{i} \cdot\right)\right\| \rightarrow 0$ for every $\varphi \in M_{*}$, there exists a bounded net $\left(z_{i}\right)_{i}$ in $\mathbb{C}$ such that $x_{i}-z_{i} 1 \rightarrow 0$ in the strong operator topology. If $M$ is a $\mathrm{II}_{1}$-factor, then by Corollary 3.8 of Co74] this is equivalent to the negation of the Murray-von Neumann property $\Gamma$. By [Ma18b, Theorem A], a factor $M$ is full if and only if $C_{\lambda \cdot \rho}^{*}(M)$ contains the algebra $\mathcal{K}\left(\mathrm{L}^{2}(M)\right)$ of compact operators. If $M$ has separable predual, $M$ is full if and only if the group of inner automorphisms $\operatorname{Inn}(M)$ is closed in $\operatorname{Aut}(M)$ Co74.

\section{Proof of the main theorem}

Our proof of Theorem $\mathrm{A}$ is based on a reduction to the following theorem of Pimsner and Popa which solves the finite index case. The second part of this statement follows implicitly from the proof of [PiPo86, Proposition 1.11].

Theorem 3.1 ( PiPo86, Proposition 1.11]). Let $M$ be a $\mathrm{II}_{1}$ factor and $N \subset$ $M$ an irreducible subfactor with finite index. Then $M$ is full if and only if $N$ is full and in that case, we have $N^{\prime} \cap M^{\omega}=\mathbf{C}$ for any ultrafilter $\omega \in \beta \mathbf{N} \backslash \mathbf{N}$.

In order to reduce the problem to the finite index case, we will need few lemmas. The first is the following bimodule interpretation of fullness.

Proposition 3.2. Let $M$ be a full factor. Then every $M-M$-bimodule that is weakly equivalent to $\mathrm{L}^{2}(M)$ must contain $\mathrm{L}^{2}(M)$.

Proof. Suppose that $M$ is full. Let $\mathcal{H}$ be an $M$ - $M$-bimodule with its binormal representation $\pi: M \odot M^{\mathrm{op}} \rightarrow \mathbf{B}(\mathcal{H})$. Suppose that $\mathcal{H}$ is weakly equivalent to $\mathrm{L}^{2}(M)$. This means that $\pi$ extends to an isometric $*$-representation $\pi: C_{\lambda \cdot \rho}^{*}(M) \rightarrow \mathbf{B}(\mathcal{H})$. Take a unit vector $\xi \in \mathrm{L}^{2}(M)$. In order to show that $\mathrm{L}^{2}(M) \subset \mathcal{H}$, it is enough to find a unit vector $\eta \in \mathcal{H}$ such that $\langle a \xi b, \xi\rangle=\langle a \eta b, \eta\rangle$ for all $a, b \in M$. Let $p$ be the projection on the one-dimensional space spanned by $\xi$. By [Co75, Theorem 2.1] and [Ma18b, Theorem A], $C_{\lambda \cdot \rho}^{*}(M)$ contains all the compact operators of $\mathrm{L}^{2}(M)$ and in particular, it contains $p$. Since $\pi$ is isometric, $\pi(p)$ is a non-zero projection in $\mathbf{B}(\mathcal{H})$ and any unit vector $\eta$ in the range of $\pi(p)$ satisfies the desired equality.

Remark 3.3. The converse of Proposition 3.2 is also true when $M$ has separable predual. Indeed, in that case, if $M$ is not full, then there exists $\theta \in \overline{\operatorname{Inn}}(M) \backslash \operatorname{Inn}(M)$. Then the correspondence $\mathrm{L}^{2}(\theta)$ is weakly equivalent to $\mathrm{L}^{2}(M)$ but does not contain it.

In the proof of our main theorem, the following key lemma will be combined with Proposition 3.2 in order to obtain the condition in Lemma 2.1.(i). 
Lemma 3.4. Let $(M, \tau)$ be a tracial von Neumann algebra. Suppose that $P \subset M$ is a subalgebra which satisfies $P=\left(P^{\prime} \cap M^{\omega}\right)^{\prime} \cap M$ for some ultrafilter $\omega \in \beta \mathbf{N} \backslash \mathbf{N}$. Then ${ }_{M} \mathrm{~L}^{2}(\langle M, P\rangle)_{M} \prec{ }_{M} \mathrm{~L}^{2}(M)_{M}$.

Proof. Let $\left\{x_{1}, \ldots, x_{n}\right\}$ be a finite subset of $M$. We will use the notations $\underline{M}=M^{\oplus n}, \underline{x}=\left(x_{1}, \ldots, x_{n}\right) \in \underline{M}, \underline{M}^{\omega}=\left(M^{\omega}\right)^{\oplus n}=\left(M^{\oplus n}\right)^{\omega}$. For every finite set $F \subset P$ and every $\varepsilon>0$, we define

$$
U_{F, \varepsilon}=\left\{u \in \mathcal{U}(M) \mid \sum_{a \in F}\|u a-a u\|_{2}<\varepsilon\right\}
$$

and we let

$$
C_{F, \varepsilon}=\overline{\operatorname{conv}}\left\{u \underline{x} u^{*} \mid u \in U_{F, \varepsilon}\right\}, \quad C=\bigcap_{F, \varepsilon} C_{F, \varepsilon} .
$$

Then $C$ is a non-empty convex weak ${ }^{*}$-compact subset of $M$. Let $y=$ $\left(y_{1}, \ldots, y_{n}\right) \in C$ be the unique element with minimal $\|\cdot\|_{2}$-norm. Observe that for every $u \in \mathcal{U}\left(P^{\prime} \cap M^{\omega}\right)$, we have $\mathrm{E}\left(u y u^{*}\right) \in C$ where $\mathrm{E}: \underline{M}^{\omega} \rightarrow \underline{M}$ is the canonical conditional expectation. Moreover, we have $\left\|\mathrm{E}\left(u y u^{*}\right)\right\|_{2} \leq$ $\left\|u \underline{y} u^{*}\right\|_{2}=\|\underline{y}\|_{2}$. Therefore, by minimality of $\|\underline{y}\|_{2}$, we must have $\underline{y}=$ $u \underline{y} \bar{u}^{*}=\mathrm{E}\left(u \underline{y} \bar{u}^{*}\right)$. This shows that $\underline{y} \in\left(P^{\prime} \cap M^{\bar{\omega}}\right)^{\prime} \cap \underline{M}=\underline{P}$. But since $\underline{y} \in C$, we also have $\mathrm{E}_{P}\left(y_{i}\right)=\mathrm{E}_{P}\left(x_{i}\right)$ for all $i \leq n$. We conclude that $y_{i}=\mathrm{E}_{P}\left(x_{i}\right)$ for all $i \leq n$. This shows that $\mathrm{E}_{P}$ is the pointwise weak*-limit of of convex combinations of inner automorphisms.

Now, $\mathrm{L}^{2}(\langle M, P\rangle)$ contains a natural $M$ - $M$-cyclic vector $\xi$ which satisfies $\langle x \xi y, \xi\rangle=\tau\left(x \mathrm{E}_{P}(y)\right)$ for all $x, y \in M$. But we have proved that $\tau\left(x \mathrm{E}_{P}(y)\right)$ can be approximated by convex combinations of $\tau\left(x u y u^{*}\right)=\langle x(u \hat{1}) y,(u \hat{1})\rangle$ where $\hat{1} \in \mathrm{L}^{2}(M)$ and $u \in \mathcal{U}(M)$. Thus ${ }_{M} \mathrm{~L}^{2}(\langle M, P\rangle)_{M} \prec{ }_{M} \mathrm{~L}^{2}(M)_{M}$.

Finally, we will need the following proposition for the reduction to the tracial case.

Proposition 3.5. Let $N \subset M$ be an inclusion of von Neumann algebras with a faithful normal conditional expectation $\mathrm{E}_{N}: M \rightarrow N$ and let $c(N) \subset$ $c(M)$ be the associated inclusion of continuous cores. If $N \subset M$ is coamenable then $c(N) \subset c(M)$ is also co-amenable.

Proof. Since $N \subset M$ is co-amenable and $M \subset c(M)$ is co-amenable by Theorem 2.3, we know that $N \subset c(M)$ is co-amenable. Since $N \subset c(N) \subset$ $c(M)$, it follows that $c(N) \subset c(M)$ is co-amenable.

Proof of Theorem $A$. We first deal with the case where $M$ is a $\mathrm{II}_{1}$ factor. Fix a free ultrafilter $\omega \in \beta \mathbf{N} \backslash \mathbf{N}$. Let $P=\left(N^{\prime} \cap M^{\omega}\right)^{\prime} \cap M$. Then we have $P=\left(P^{\prime} \cap M^{\omega}\right)^{\prime} \cap M$. By Lemma 3.4, we have ${ }_{M} \mathrm{~L}^{2}(\langle M, P\rangle)_{M} \prec{ }_{M} \mathrm{~L}^{2}(M)_{M}$. Since $N \subset M$ is co-amenable and $N \subset P$, we have that $P \subset M$ is still coamenable. Therefore ${ }_{M} \mathrm{~L}^{2}(M)_{M} \prec{ }_{M} \mathrm{~L}^{2}(\langle M, P\rangle)_{M}$. This shows that the $M$ $M$-bimodule ${ }_{M} \mathrm{~L}^{2}(\langle M, P\rangle)_{M}$ is weakly equivalent to ${ }_{M} \mathrm{~L}^{2}(M)_{M}$. Therefore, by Proposition 3.2, we have ${ }_{M} \mathrm{~L}^{2}(M)_{M} \subset{ }_{M} \mathrm{~L}^{2}(\langle M, P\rangle)_{M}$. Therefore, by Lemma 2.1, there exists a non-zero $p \in P^{\prime} \cap M$ such that $P p \subset p M p$ is an irreducible subfactor with finite index. Since $p M p$ is full, we obtain $p\left(P^{\prime} \cap M^{\omega}\right) p=\mathbf{C} p$ by Theorem 3.1. In particular, we have $p \in N^{\prime} \cap M$ and $p\left(N^{\prime} \cap M^{\omega}\right) p=\mathbf{C} p$. 
Now suppose that $M$ is of arbitrary type. Let $K$ be a full type $\mathrm{III}_{1}$ factor with separable predual and with trivial $\tau$-invariant (for example take $K$ the free Araki-Woods factor associated to the regular orthogonal representation of $\mathbf{R}$, see [Sh98]). Let $M_{1}=M \bar{\otimes} K$ and $N_{1}=N \bar{\otimes} K$. Then by [HMV16, $M_{1}$ is a full type $\mathrm{III}_{1}$ factor with trivial $\tau$-invariant. Therefore by [Ma16], we know that the continuous core $c\left(M_{1}\right)$ is a full $\mathrm{II}_{\infty}$ factor. Now, pick a faithful normal state $\varphi$ on $N$ and extend it to $M$ by using a faithful normal conditional expectation $\mathrm{E}_{N}: M \rightarrow N$. Define a faithful normal state on $M_{1}$ by $\varphi_{1}=\varphi \otimes \psi$ where $\psi$ is any faithful normal state on $K$. Then we have a trace preserving inclusion of the continuous cores $c\left(N_{1}\right)=N_{1} \rtimes_{\sigma^{\varphi_{1}}} \mathbf{R} \subset$ $c\left(M_{1}\right)=M_{1} \rtimes_{\sigma^{\varphi_{1}}} \mathbf{R}$. The inclusion $c\left(N_{1}\right) \subset c\left(M_{1}\right)$ is co-amenable by Proposition 3.5. Pick a non-zero finite trace projection $q \in L(\mathbf{R})=\mathbf{C} \rtimes_{\varphi}$ $\mathbf{R} \subset c\left(N_{1}\right)$. Observe that the central support of $q$ in $c\left(N_{1}\right)$ is 1 because $N_{1}$ is of type $\mathrm{III}_{1}$. Therefore $q c\left(N_{1}\right) q$ is still a co-amenable subalgebra in the full $\mathrm{II}_{1}$ factor $q c\left(M_{1}\right) q$. Thus, by the first part of the proof, there exists a nonzero projection $p \in c\left(N_{1}\right)^{\prime} \cap c\left(M_{1}\right)$ such that $p q\left(c\left(N_{1}\right)^{\prime} \cap c\left(M_{1}\right)^{\omega}\right) p q=\mathbf{C} p q$. Since $q$ has central support 1 in $c\left(N_{1}\right)$, this implies $p\left(c\left(N_{1}\right)^{\prime} \cap c\left(M_{1}\right)^{\omega}\right) p=\mathbf{C} p$. Now, observe that $c\left(N_{1}\right)^{\prime} \cap c\left(M_{1}\right)=N^{\prime} \cap M_{\varphi}$ because $K$ is of type $\mathrm{III}_{1}$. Moreover, $N^{\prime} \cap M_{\varphi^{\omega}}^{\omega} \subset c\left(N_{1}\right)^{\prime} \cap c\left(M_{1}\right)^{\omega}$. Therefore, we have $p \in N^{\prime} \cap M_{\varphi}$ and $p\left(N^{\prime} \cap M_{\varphi^{\omega}}^{\omega}\right) p=\mathbf{C} p$. Since $p\left(N^{\prime} \cap M_{\varphi^{\omega}}^{\omega}\right) p$ is the centralizer of the state $\varphi^{\omega}$ restricted to $p\left(N^{\prime} \cap M^{\omega}\right) p$, we know by [AH12, Lemma 5.3] that $p\left(N^{\prime} \cap M^{\omega}\right) p$ is either trivial or a type $\mathrm{III}_{1}$ factor. But the latter case cannot happen because if $p\left(N^{\prime} \cap M^{\omega}\right) p$ is of type $\mathrm{III}_{1}$, then for any $\varepsilon>0$, we can find a Haar unitary $u \in p\left(N^{\prime} \cap M^{\omega}\right) p$ such that $\left\|u \varphi^{\omega}-\varphi^{\omega} u\right\| \leq \varepsilon$ and by a diagonal extraction, this would imply that $p\left(N^{\prime} \cap M_{\varphi^{\omega}}^{\omega}\right) p \neq \mathbf{C}$. Thus we conclude that $p\left(N^{\prime} \cap M^{\omega}\right) p=\mathbf{C} p$.

Remark 3.6. Note that the w-spectral gap property for an inclusion of factors is weaker than the spectral gap property in general (we refer the reader to the introduction of IV15] for the definitions and a discussion of this subtle difference). Thus, one might wonder wether in Corollary B, one can improve the w-spectral gap to a true spectral gap. The following example shows that the answer is negative in general.

Let $\mathbf{F}_{2} \curvearrowright(X, \mu)$ be a free ergodic pmp action which is weakly compact (see [OP07, Section 3]) and strongly ergodic but does not have spectral gap. The weak compactness implies that the inclusion $L\left(\mathbf{F}_{2}\right) \subset \mathrm{L}^{\infty}(X) \rtimes \mathbf{F}_{2}$ is co-amenable by [OP07, Proposition 3.2]. Since the action $\mathbf{F}_{2} \curvearrowright(X, \mu)$ is strongly ergodic and $\mathbf{F}_{2}$ is non-inner amenable, the crossed product $\mathrm{L}^{\infty}(X) \rtimes$ $\mathbf{F}_{2}$ is full by Ch81. However, since $\mathbf{F}_{2} \curvearrowright(X, \mu)$ does not have spectral gap, the subfactor $L\left(\mathbf{F}_{2}\right)$ does not have spectral gap in $\mathrm{L}^{\infty}(X) \rtimes \mathbf{F}_{2}$ (see the introduction of [IV15]).

In order to construct such an action $\mathbf{F}_{2} \curvearrowright(X, \mu)$, one can use the result [HK05, Theorem A.3.2] which was pointed to us by Adrian Ioana. We start from a compact free ergodic pmp action $\mathbf{F}_{2}=\langle a, b\rangle \curvearrowright(Y, \nu)$ which has spectral gap and such that $a$ acts ergodically on $(Y, \nu)$. Then we use [HK05, Theorem A.3.2] to obtain a new action $\mathbf{F}_{2} \curvearrowright(X, \mu)$ which is orbit equivalent to $\mathbf{F}_{2} \curvearrowright(Y, \nu)$ but does not have spectral gap. This new action $\mathbf{F}_{2} \curvearrowright(X, \mu)$ is strongly ergodic and also weakly compact by [Io08, Section $6]$, as we wanted. 


\section{Actions of COMPaCt GROUPS ON FUll FACTORS}

Recall that an action $\sigma: G \curvearrowright M$ of a locally compact group $G$ on a factor $M$ is called outer if $\sigma_{g} \notin \operatorname{Inn}(M)$ for all $g \in G \backslash\{1\}$. It is called strictly outer if $M^{\prime} \cap\left(M \rtimes_{\sigma} G\right)=\mathbf{C}$. Finally, it is called minimal if it is faithful and $\left(M^{G}\right)^{\prime} \cap M=$ C. A minimal action is strictly outer and a strictly outer action is outer, but the reverse implications are not true in general. When $G$ is discrete, $\sigma$ is outer if and only if it is strictly outer. When $G$ is compact, $\sigma$ is minimal if and only if it is strictly outer. We refer the reader to Va05. for further details regarding these notions.

In order to prove Theorem [C, we will first need to prove the following dichotomy:

Theorem 4.1. Let $\sigma: G \curvearrowright M$ be an action of a compact group $G$ on a factor $M$. Suppose that $\sigma$ is outer. Then $\left(M^{G}\right)^{\prime} \cap M$ is either trivial or diffuse.

The following lemma is an easy consequence of the Peter-Weyl theorem.

Lemma 4.2. Let $M$ be a von Neumann algebra. The following are equivalent:

(i) $M$ is a multi-matrix algebra, i.e. $M \cong \bigoplus_{i \in I} M_{n_{i}}$ (C) for some (possibly infinite) family of integers $n_{i} \geq 1, i \in I$.

(ii) The unitary group of $M$ is compact.

(iii) $M$ is generated by a compact group of unitaries.

Lemma 4.3. Let $\sigma: G \curvearrowright M$ be an action of a compact group $G$ on a factor $M$. Then the following are equivalent.

(i) $M^{\prime} \cap(M \rtimes G)$ is a multi-matrix algebra.

(i) $\left(M^{G}\right)^{\prime} \cap M$ is a multi-matrix algebra.

(ii) $M^{\prime} \cap(M \rtimes G)$ is completely atomic.

(ii) $^{\prime}\left(M^{G}\right)^{\prime} \cap M$ is completely atomic.

(iii) $M^{\prime} \cap(M \rtimes G)$ is not diffuse.

(iii) ${ }^{\prime}\left(M^{G}\right)^{\prime} \cap M$ is not diffuse.

Proof. We will fix the notation $N=M \bar{\otimes} \mathbf{B}\left(\mathrm{L}^{2}(G)\right)$ and consider the action $\sigma \otimes \rho: G \curvearrowright N$ where $\rho: G \curvearrowright \mathbf{B}\left(\mathrm{L}^{2}(G)\right)$ is the action coming from the right regular representation as in the preliminary section. Recall that we have an isomorphism $\pi: M \rtimes_{\sigma} G \rightarrow N^{G}$ which extends to an isomorphism from $\left\langle M \rtimes_{\sigma} G, M\right\rangle$ to $N$. Since $G$ is compact, we also observe that if $e_{G} \in$ $\mathbf{B}\left(\mathrm{L}^{2}(G)\right)$ is the rank-one projection on the scalar functions, then $e_{G}$ is invariant under $\rho$ and $\sigma \otimes \rho$ restricts to $\sigma$ on $e_{G} N e_{G}=M$.

The implications (i) $\Rightarrow$ (ii) $\Rightarrow$ (iii) and (i) ${ }^{\prime} \Rightarrow(\text { ii })^{\prime} \Rightarrow(\text { iii) })^{\prime}$ are obvious. The implications (i) $\Rightarrow(\text { i })^{\prime}$, (ii) $\Rightarrow$ (ii) $^{\prime}$ and (iii) ${ }^{\prime} \Rightarrow$ (iii) are also easy. Indeed, this follows from [Pa77, Theorem 4.2] which says that there is a surjective *-homomorphism from $M \rtimes G$ onto $\left\langle M, M^{G}\right\rangle$ which is the identity on $M$. Hence $M^{\prime} \cap(M \rtimes G)$ surjects onto $M^{\prime} \cap\left\langle M, M^{G}\right\rangle \cong\left(\left(M^{G}\right)^{\prime} \cap M\right)^{\text {op }}$. We conclude that if $M^{\prime} \cap(M \rtimes G)$ is a multi-matrix algebra (resp. completely atomic, resp. diffuse) then $\left(M^{G}\right)^{\prime} \cap M$ is also a multi-matrix algebra (resp. completely atomic, resp. diffuse).

$(\mathrm{i})^{\prime} \Rightarrow$ (i). Observe that $\left(M^{G}\right)^{\prime} \cap(M \rtimes G)=\left(\left(M^{G}\right)^{\prime} \cap M\right) \rtimes G$ (this follows from the fact that $\left.\pi\left(M^{G}\right)=M^{G} \otimes \mathbf{C} \subset M \bar{\otimes} \mathbf{B}\left(\mathrm{L}^{2}(G)\right)\right)$. Since 
$\left(M^{G}\right)^{\prime} \cap M$ is a multi-matrix algebra then its unitary group $\mathcal{U}\left(\left(M^{G}\right)^{\prime} \cap M\right)$ is compact. Therefore, $\left.\left(M^{G}\right)^{\prime} \cap M\right) \rtimes G$ is generated by the compact group $\mathcal{U}\left(\left(M^{G}\right)^{\prime} \cap M\right) \rtimes G$, hence $\left(M^{G}\right)^{\prime} \cap(M \rtimes G)$ is a multi-matrix algebra. In particular $M^{\prime} \cap(M \rtimes G)$ is a multi-matrix algebra.

$\left(\right.$ ii $^{\prime} \Rightarrow(\mathrm{i})^{\prime}$. This can be deduced from [HLS81] but we provide an easy proof in our specific situation. Take $p$ a minimal projection in the center of $M^{G}$. Then the action of $G$ on the algebra $\left.D=\left(\left(M^{G}\right)^{\prime} \cap M\right)\right) p$ is ergodic, i.e. $D^{G}=$ C. Since $D$ is completely atomic, there exists a unique faithful semifinite trace $\tau$ on $D$ such that $\tau(e)=1$ for every minimal projection $e \in D$. Since $\tau$ is unique, it must be $G$-invariant. But the canonical $G$ invariant conditional expectation $E: M \rightarrow M^{G}$ already restricts to a $G$ invariant state $\psi$ on $D$. Since $D^{G}=\mathbf{C}$, this forces $\tau$ to be proportional to $\psi$. In particular, $\tau$ is finite and $D$ must be finite dimensional. This holds for every minimal projection $p \in \mathcal{Z}\left(M^{G}\right)$ hence $\left(M^{G}\right)^{\prime} \cap M$ is a direct sum of finite dimensional algebras.

(iii) ${ }^{\prime} \Rightarrow$ (ii). Let $z$ be the maximal central projection in $\left(M^{G}\right)^{\prime} \cap M$ such that $\left(\left(M^{G}\right)^{\prime} \cap M\right) z$ is completely atomic. Then $z$ must be $G$-invariant, hence $z \in \mathcal{Z}\left(M^{G}\right)$. Consider the truncated action $\sigma^{\prime}: G \curvearrowright Q=z M z$. Then $\left(Q^{G}\right)^{\prime} \cap Q$ is completely atomic. Hence, by (ii) $\Rightarrow(\text { i })^{\prime} \Rightarrow$ (i) $\Rightarrow$ (ii), we have that $Q^{\prime} \cap(Q \rtimes G)$ is completely atomic. But we have $Q^{\prime} \cap(Q \rtimes G)=$ $\left(M^{\prime} \cap(M \rtimes G)\right) z$ and $\left(M^{\prime} \cap(M \rtimes G)\right) z$ is isomorphic to $M^{\prime} \cap(M \rtimes G)$ because $z \in M$ and $M$ is a factor. Therefore $M^{\prime} \cap(M \rtimes G)$ is completely atomic.

(iii) $\Rightarrow$ (ii). If $M^{\prime} \cap(M \rtimes G)$ is not diffuse, then $\left(N^{G}\right)^{\prime} \cap N$, which is antiisomorphic to it, is also not diffuse. Then the implications (iii) ${ }^{\prime} \Rightarrow$ (ii) $\Rightarrow$ (ii) show that $\left(N^{G}\right)^{\prime} \cap N$ is completely atomic, hence $M^{\prime} \cap(M \rtimes G)$ is completely atomic.

The reader may now check that all the implications we proved form a connected graph, so that all the properties are equivalent.

Proof of Theorem 4.1. Suppose that $\left(M^{G}\right)^{\prime} \cap M$ is not diffuse. By Lemma 4.3. we know that $\left(M^{G}\right)^{\prime} \cap M$ is a multi-matrix algebra. We want to show that $\sigma$ is minimal, i.e. that $\left(M^{G}\right)^{\prime} \cap M=\mathbf{C}$.

We first deal with the case where $\left(M^{G}\right)^{\prime} \cap M$ is a factor. In this case, since $Q=\left(M^{G}\right)^{\prime} \cap M$ is a finite dimensional factor, we have $M=M_{0} \otimes Q$ where $M_{0}=Q^{\prime} \cap M$. Observe that the action $\sigma_{0}: G \curvearrowright M_{0}$ is minimal. Indeed, by construction we have $M^{G}=\left(M_{0}\right)^{G}$ and $\left(M^{G}\right)^{\prime} \cap M_{0}=\mathbf{C}$, and $\sigma_{0}$ is outer (hence faithful) because $\sigma$ is outer. Now, suppose that $Q \neq \mathbf{C}$ and consider the representation of $G$ on $Q \ominus \mathbf{C}$ induced by the action $G \curvearrowright Q$. Choose $V \subset Q \ominus \mathbf{C}$ an irreducible subrepresentation. Since $\sigma_{0}$ is minimal, we can find a copy of the contragredient representation $\bar{V} \subset M_{0}$. Then $\bar{V} \otimes V \subset M_{0} \otimes(Q \ominus \mathbf{C})=M \ominus M_{0}$ contains a copy of the trivial representation of $G$. But this contradicts the fact that $M^{G} \subset M_{0}$. We conclude that $Q=\mathbf{C}$, hence $\sigma$ is minimal.

Now, we deal with the general case where $\left(M^{G}\right)^{\prime} \cap M$ is not necessarily a factor. Among all open subgroups $K \subset G$ and all non-zero projections $e \in M^{K}$, we choose a pair $(K, e)$ such that the dimension of $e\left(\left(M^{K}\right)^{\prime} \cap M\right) e$ is minimal. Take $f$ a minimal projection in the center of $e\left(\left(M^{K}\right)^{\prime} \cap M\right) e$. Then there exists an open subgroup $H \subset K$ which stabilizes $f$. Since 
$f\left(\left(M^{H}\right)^{\prime} \cap M\right) f \subset e\left(\left(M^{K}\right)^{\prime} \cap M\right) e$, by minimality of $(K, e)$, we must have $f\left(\left(M^{H}\right)^{\prime} \cap M\right) f=e\left(\left(M^{K}\right)^{\prime} \cap M\right) e$ hence $f=e$. This shows that $e\left(\left(M^{K}\right)^{\prime} \cap\right.$ $M) e=\left((e M e)^{K}\right)^{\prime} \cap e M e$ is a factor. But the action of $K$ on $e M e$ is outer (because $K \curvearrowright M$ is outer and $M$ is a factor). Since we already treated the factorial case, we get that $K \curvearrowright e M e$ is minimal. Therefore $K \curvearrowright e M e$ is strictly outer, i.e. $\left(M^{\prime} \cap(M \rtimes K)\right) e=(e M e)^{\prime} \cap(e M e \rtimes K)=\mathbf{C} e$. Since $M$ is a factor, this implies that $M^{\prime} \cap(M \rtimes K)=\mathbf{C}$, i.e. $K \curvearrowright M$ is strictly outer. Finally, using the fact that $K$ is open in $G$, we will conclude that $G \curvearrowright M$ is also strictly outer, hence minimal. Indeed, since $K$ is open, any $x \in M \rtimes G$ has a "Fourier decomposition" $x=\sum_{g \in G / K} u_{g} x_{g}$ where $x_{g} \in M \rtimes K$ (we implicitly choose a set of representatives of each equivalence class in $G / K$ ). We refer to [BB16] for an explanation of this "Fourier decomposition". If $x \in M^{\prime} \cap(M \rtimes G)$, we must have $x_{g} a=\sigma_{g}^{-1}(a) x_{g}$ for all $a \in M$. By [BB16, Corollary 3.11], and since $\sigma$ is outer, this is only possible if $g \in K$. Therefore $M^{\prime} \cap(M \rtimes G)=M^{\prime} \cap(M \rtimes K)=\mathbf{C}$ as we wanted.

Remark 4.4. Let $\sigma: G \curvearrowright M$ be an action of a compact group $G$ on a factor $M$. Let $H=\left\{g \in G \mid \sigma_{g}\right.$ is inner $\}$. Then one can deduce from Theorem 4.1 that either $\left(M^{G}\right)^{\prime} \cap M$ is diffuse or

$$
\left(M^{G}\right)^{\prime} \cap M=\left(M^{H}\right)^{\prime} \cap M=\left\{u \in M \mid \exists g \in G, \operatorname{Ad}(u)=\sigma_{g}\right\}^{\prime \prime} .
$$

Proof of Theorem $\square$. Suppose first that $M^{G} \subset M$ is co-amenable. Since $M$ is full, then by Theorem $\mathrm{A}$, $\left(M^{G}\right)^{\prime} \cap M$ is not diffuse and by combining with Theorem 4.1 it must be trivial. We conclude that $\left(M^{G}\right)^{\prime} \cap M^{\omega}=\mathbf{C}$ by Theorem A. Next, since we have $\pi(M \rtimes G)$ is with expectation in $M \bar{\otimes}$ $\mathbf{B}\left(\mathrm{L}^{2}(G)\right)$, we have $\pi(M \rtimes G)^{\omega} \subset M^{\omega} \bar{\otimes} \mathbf{B}\left(\mathrm{L}^{2}(G)\right)$. Since $\pi\left(M^{G}\right)=M^{G} \otimes \mathbf{C}$, we get $\pi\left(M^{\prime} \cap(M \rtimes G)^{\omega}\right) \subset\left(\left(M^{G}\right)^{\prime} \cap M^{\omega}\right) \bar{\otimes} \mathbf{B}\left(\mathrm{L}^{2}(G)\right)=\mathbf{C} \otimes \mathbf{B}\left(\mathrm{L}^{2}(G)\right)$. We conclude that $M^{\prime} \cap(M \rtimes G)^{\omega}=M^{\prime} \cap(M \rtimes G)=\mathbf{C}$.

Now, in the general case, consider the action $\sigma \otimes \rho: G \curvearrowright N=M \bar{\otimes}$ $\mathbf{B}\left(\mathrm{L}^{2}(G)\right)$. Then the inclusion $N^{G} \subset N$ which is isomorphic to $M \rtimes G \subset$ $\langle M \rtimes G, M\rangle$ is co-amenable by Theorem 2.3. So we can apply the first case to deduce that $\sigma \otimes \rho$ is minimal, hence $\sigma$ is also minimal. In particular, $M^{G} \subset M$ is co-amenable and we conclude by the first case.

\section{Appendix A. Bimodule ChaRACTERIZATION OF CO-AMENABILITY}

Let $M$ be a von Neumann algebra. We will denote by $\mathrm{L}^{2}(M)$ the standard Hilbert space for $M$, by $J_{M}$ the modular conjugation, by $\mathrm{L}^{2}(M)^{+}$the natural positive cone, and by $\pi_{M}: M \odot M^{\mathrm{op}} \rightarrow \mathbf{B}\left(\mathrm{L}^{2}(M)\right)$ the natural binormal *representation. We define the norm

$$
M \odot M^{\mathrm{op}} \ni T \mapsto\|T\|_{\pi_{M}}=\left\|\pi_{M}(T)\right\|_{\mathbf{B}\left(\mathrm{L}^{2}(M)\right)} .
$$

For $x \in M$, we will use the notation $\bar{x}=\left(x^{*}\right)^{\mathrm{op}}=\left(x^{\mathrm{op}}\right)^{*} \in M^{\mathrm{op}}$.

Haagerup has made a deep study of the norm

$$
M^{\oplus n} \ni\left(x_{1}, \ldots, x_{n}\right) \mapsto\left\|\sum_{i} x_{i} \otimes \overline{x_{i}}\right\|_{\pi_{M}}
$$

and obtained many remarkable results in Ha93, but we need only the following (Theorem 2.1 in Ha93]). This was proved earlier in the semifinite 
case by Pisier (Theorem 2.1 in [Pi95]). Alternative proofs are found in [JP10] and $\mathrm{Xu05}$.

Theorem A.1 (Pisier-Haagerup). For any von Neumann algebra $M$ and any $x_{1}, \ldots, x_{n} \in M$,

$$
\left\|\sum_{i} x_{i} \otimes \overline{x_{i}}\right\|_{\pi_{M}}^{1 / 2}=\left\|\left(x_{1}, \ldots, x_{n}\right)\right\|_{1 / 2},
$$

where the latter norm is the complex interpolation of equivalent norms $\|\cdot\|_{0}$ and $\|\cdot\|_{1}$ on $M^{\oplus n}$ that are given by

$$
\left\|\left(a_{1}, \ldots, a_{n}\right)\right\|_{0}:=\left\|\sum_{i} a_{i} a_{i}^{*}\right\|^{1 / 2} \text { and }\left\|\left(a_{1}, \ldots, a_{n}\right)\right\|_{1}:=\left\|\sum_{i} a_{i}^{*} a_{i}\right\|^{1 / 2} .
$$

In particular, for any (not necessarily normal) unital completely positive map $\theta$ from $M$ into a von Neumann algebra $N$ and any $x_{1}, \ldots, x_{n} \in M$,

$$
\left\|\sum_{i} \theta\left(x_{i}\right) \otimes \overline{\theta\left(x_{i}\right)}\right\|_{\pi_{N}} \leq\left\|\sum_{i} x_{i} \otimes \overline{x_{i}}\right\|_{\pi_{M}} .
$$

Note that the second half of Theorem A.1 is a trivial consequence of the interpolation theorem, because $\theta \otimes \mathrm{id}_{n}:\left(M^{\oplus n},\|\cdot\|_{t}\right) \rightarrow\left(N^{\oplus n},\|\cdot\|_{t}\right)$ is contractive at $t=0,1$. The purpose of this appendix is to give a proof of the following corollary to Theorem A.1.

Corollary A.2 (cf. Corollary 3.8 in [Ha93]). For von Neumann algebras $N \subset M$, the following are equivalent.

(i) There is a conditional expectation of $M$ onto $N$.

(ii) For every $x_{1}, \ldots, x_{n} \in N$,

$$
\left\|\sum_{i} x_{i} \otimes \overline{x_{i}}\right\|_{\pi_{N}}=\left\|\sum_{i} x_{i} \otimes \overline{x_{i}}\right\|_{\pi_{M}} .
$$

(iii) ${ }_{N} \mathrm{~L}^{2}(N)_{N} \prec{ }_{N} \mathrm{~L}^{2}(M)_{N}$. Namely, for every $x_{i}, y_{i} \in N$,

$$
\left\|\sum_{i} x_{i} \otimes \overline{y_{i}}\right\|_{\pi_{N}} \leq\left\|\sum_{i} x_{i} \otimes \overline{y_{i}}\right\|_{\pi_{M}} .
$$

The following reformulation of Corollary A.2 closes the circle of implications of Propositions 2.5 in AD95.

Corollary A.3 (cf. Proposition 3.6 in [AD95]). Let ${ }_{M} \mathcal{H}_{N}$ be an $M-N$ correspondence and put $N_{1}=\mathbf{B}_{N^{\text {op }}}(\mathcal{H})$, the commutant of the right $N$-action. Then the following are equivalent.

(i) ${ }_{M} \mathcal{H}_{N}$ is left-amenable, i.e. ${ }_{M} \mathrm{~L}^{2}(M)_{M} \prec{ }_{M} \mathrm{~L}^{2}\left(N_{1}\right)_{M}$.

(ii) There is a conditional expectation of $N_{1}$ onto $M$.

We recall the notion of the selfpolar forms (Co74b, Wo74), which plays a central role in Ha93] as well as in the proof of Corollary A.2. Associated with any normal state $\varphi$ on $M$, there are a canonical unit vector $\xi_{\varphi}$ in $\mathrm{L}^{2}(M)^{+}$and the selfpolar form $s_{\varphi}: M \times M \rightarrow \mathbb{C}$ given by

$$
s_{\varphi}(x, y)=\left\langle\pi_{M}(x \otimes \bar{y}) \xi_{\varphi}, \xi_{\varphi}\right\rangle_{\mathrm{L}^{2}(M)} .
$$

Proposition A.4 (Theorems 1.1 and 2.1 in Wo74]). The selfpolar form $s_{\varphi}$ satisfies the following properties.

(i) $s_{\varphi}$ is a positive semi-definite sesqui-linear form on $M \times M$, 
(ii) $s_{\varphi} \geq 0$ on $M_{+} \times M_{+}$,

(iii) $s_{\varphi}(x, 1)=\varphi(x)$ for $x \in M$, and

(iv) $s_{\varphi}$ is selfpolar.

Moreover, if $s$ is any sesqui-linear form on $M$ which satisfies (i) - (iii) above (with $s_{\varphi}$ exchanged by $s$ ), then $s(x, x) \leq s_{\varphi}(x, x)$ for every $x \in M$.

We do not introduce the selfpolar property (iv), since we will not use it (in an explicit way). We use the following well-known variant of the HahnBanach theorem.

Lemma A.5. Let $C$ be a convex cone of a real vector space $V, p: V \rightarrow \mathbf{R} a$ sublinear function, and $q: C \rightarrow \mathbf{R}$ a superlinear function. This means that $p(x+y) \leq p(x)+p(y)$ and $p(\lambda x)=\lambda p(x)$ for every $x, y \in V$ and $\lambda \in \mathbf{R}_{\geq 0}$; and that $-q$ is sublinear. If $q \leq p$ on $C$, then there is a linear functional $\psi$ on $V$ such that $\psi \leq p$ on $V$ and $q \leq \psi$ on $C$.

Proof. Observe that $r(x):=\inf \{p(x+y)-q(y): y \in C\}$ is a sublinear function on $V$ such that $-p(-x) \leq r(x) \leq p(x)$ for $x \in V$ (in particular $r$ takes values in $\mathbf{R}$ ). By the Hahn-Banach theorem, there is a linear functional $\psi$ on $V$ such that $\psi \leq r$. One has $-\psi(x) \leq r(-x) \leq-q(x)$ for $x \in C$.

Proof of Corollary A.2. The equivalence (ii) $\Leftrightarrow$ (ii) is proved in Corollary 3.8 in [Ha93. That (ii) $\Rightarrow$ (iii) follows from Theorem A.1. That (iii) $\Rightarrow$ (ii) is proved in Proposition 2.5 in [AD95. Thus it is left to show (iii) $\Rightarrow$ (iii). We closely follow the proof of Corollary 3.8 (Theorem 3.7) in Ha93. Take any normal state $\varphi$ on $N$ and consider the convex cone $C:=\left\{\sum_{i} x_{i} \otimes \overline{x_{i}}: x_{i} \in\right.$ $N\} \subset M \odot M^{\text {op }}$ and the seminorm $p(\cdot)=\left\|\pi_{M}(\cdot)\right\|_{\mathbf{B}\left(\left(\mathrm{L}^{2}(M)\right)\right.}$ on $M \odot M^{\text {op }}$. Since

$$
\sum_{i} s_{\varphi}\left(x_{i}, x_{i}\right)=\left\langle\pi_{N}\left(\sum_{i} x_{i} \otimes \overline{x_{i}}\right) \xi_{\varphi}, \xi_{\varphi}\right\rangle \leq p\left(\sum_{i} x_{i} \otimes \overline{x_{i}}\right)
$$

by condition (iii), Lemma A.5 yields $\psi \in \mathbf{B}\left(\mathrm{L}^{2}(M)\right)^{*}$ of norm 1 such that $s_{\varphi}(x, x) \leq \Re \psi\left(\pi_{M}(x \otimes \bar{x})\right)$ for $x \in N$. Observe that $\|\psi\| \leq 1$ and $1 \leq$ $\Re \psi(1)$ imply that $\psi$ is a state. The state $\psi^{*}$ on $\mathbf{B}\left(\mathrm{L}^{2}(M)\right)$ defined by $\psi^{*}(T)=\psi\left(J_{M} T^{*} J_{M}\right)$ satisfies $\psi^{*}\left(\pi_{M}(x \otimes \bar{y})\right)=\overline{\psi\left(\pi_{M}(y \otimes \bar{x})\right)}$ for every $x, y \in M$. Thus by replacing $\psi$ with the state $\frac{1}{2}\left(\psi+\psi^{*}\right)$, we may assume that $s: M \times M \ni(x, y) \mapsto \psi\left(\pi_{M}(x \otimes \bar{y})\right)$ is a sesqui-linear form such that $s_{\varphi}(x, x) \leq s(x, x)$ for every $x \in N$. Since $s_{\varphi}(1+\lambda x, 1+\lambda x) \leq s(1+\lambda x, 1+\lambda x)$ for all $\lambda \in \mathbf{R}$ and a fixed $x \in N$, one sees $\varphi(x)=s(x, 1)$ for $x \in N$. It follows from Proposition A.4 that $s_{\varphi}(x, x)=s(x, x)$ for every $x \in N$. Hence by polarization identity, $s_{\varphi}=s$ and so for every $T=\sum_{i} x_{i} \otimes \overline{y_{i}} \in N \odot N^{\text {op }}$ we have

$$
\left\langle\pi_{N}(T) \xi_{\varphi}, \xi_{\varphi}\right\rangle=\sum_{i} s_{\varphi}\left(x_{i}, y_{i}\right)=\sum_{i} s\left(x_{i}, y_{i}\right)=\psi\left(\pi_{M}(T)\right) .
$$

Since $\left\{\xi_{\varphi}\right\}_{\varphi}$ is a $\pi_{N}$-cyclic family, one obtains

$$
\left\|\pi_{N}(T)\right\|_{\mathbf{B}\left(\mathrm{L}^{2}(N)\right)} \leq\left\|\pi_{M}(T)\right\|_{\mathbf{B}\left(\mathrm{L}^{2}(M)\right)}
$$

which is precisely condition (iii). 


\section{REFERENCES}

[AD95] C. Anantharaman-Delaroche, Amenable correspondences and approximation properties for von Neumann algebras. Pacific J. of Math. 171 (1995).

[AH12] H. Ando, U. HaAgerup, Ultraproducts of von Neumann algebras. J. Funct. Anal. 266 (2014), 6842-6913

[Be90] E. BÉdos. On actions of amenable groups on $\mathrm{II}_{1}$-Factors. J. Funct. Anal. 91 (1990), 404-414.

[Bi90] D. Bisch, On the existence of central sequences in subfactors. Trans. Amer. Math. Soc. 321 (1990), 117-128.

[Bi94] D. Bisch. Central sequences in subfactors II. Proc. Amer. Math. Soc. 121 (1994), 725-731.

[BB16] A. Brothier, R. Boutonnet, Crossed products by locally compact groups: Intermediate subfactors. Journal of Operator Theory 79 (2018), 101-137.

[Ch79] M. ChodA. A characterization of crossed product actions by discrete outer automorphism groups. J. Math. Soc. Japan 31 (1979), 257-261.

[Ch81] M. ChodA, Inner amenability and fullness. Proc. Amer. Math. Soc. 86 (1982), 663-666.

[Co74] A. Connes, Almost periodic states and factors of type $\mathrm{III}_{1}$. J. Funct. Anal. 16 (1974), 415-445.

[Co74b] A. Connes, Caractérisation des espaces vectoriels ordonnés sous-jacents aux algèbres de von Neumann. Ann. Inst. Fourier (Grenoble) 24 (1974), 121-155.

[Co75] A. Connes, Classification of injective factors. Cases $\mathrm{II}_{1}, \mathrm{II}_{\infty}, \mathrm{III}_{\lambda}, \lambda \neq 1$. Ann. of Math. 74 (1976), 73-115.

[Ef73] E. G. Effros, Property $\Gamma$ and inner amenability. Proc. Amer. Math. Soc. 47 (1975), 483-486.

[Ha73] U. HAagerup, The standard form of von Neumann algebras. Math. Scand. 37 (1975), 271-283.

[Ha93] U. HAagerup, Selfpolar forms, conditional expectations and the weak expectation property for $\mathrm{C}^{*}$-algebras. Preprint. 1993.

[HK05] G. HJORTh, A. S. KeCHRIS Rigidity theorems for actions of product groups and countable Borel equivalence relations. Mem. of the Amer. Math. Soc. 833 (2005), 1-109.

[HLS81] R. Hoegh-Krohn, M. B. Landstad and E. Stormer, Compact Ergodic Groups of Automorphisms. Ann. of Math. 114 (1981), 75-86.

[HMV16] C. Houdayer, A. Marrakchi, P. Verraedt, Fullness and Connes' $\tau$ invariant of type III tensor product factors. To appear in J. Math. Pures Appl. arXiv: 1611.07914.

[Io08] A. IoAna, Cocycle Superrigidity for Profinite Actions of Property (T) Groups. Duke Math. J. 157 (2011), no. 2, 337-367

[IV15] A. IoAnA, S. VAES, Spectral gap for inclusions of von Neumann algebras. Appendix to the article Cartan subalgebras of amalgamated free product $\mathrm{II}_{1}$ factors by A. IoAnA in Ann. Sci. École Norm. Sup. 48 (2015), 71-130.

[Jo81] V.F.R. Jones, Central sequences in crossed products of full factors. Duke Math. J. 49 (1982), 29-33.

[JP10] M. Junge AND J. PARCET, Mixed-norm inequalities and operator space $L_{p}$ embedding theory. Mem. Amer. Math. Soc. 203 (2010), no. 953, vi+155 pp.

[Ma16] A. MARraKchi, Spectral gap characterization of full type III factors. To appear in J. Reine Angew. Math. arXiv:1605.09613

[Ma18] A. MARRAKCHI, Fullness of crossed products of factors by discrete groups. Preprint. arXiv: 1811.08274.

[Ma18b] A. MARRAKCHI, Full factors, bicentralizer flow and approximately inner automorphisms. Preprint. arXiv:1811.10253.

[MoPo03] N. Monod, S. PopA. On Co-amenability for Groups and von Neumann Algebras. Comptes Rendus Mathématiques de l'Académie des Sciences, La Société Royale du Canada 25, 3 (2003), 82-87. 
[MvN43] F. Murray, J. von Neumann, Rings of operators. IV. Ann. of Math. 44 (1943), 716-808.

[Oc85] A. OCNEANU, Actions of discrete amenable groups on von Neumann algebras. Lecture Notes in Mathematics, 1138. Springer-Verlag, Berlin, 1985. iv+115 pp.

[OP07] N. Ozawa, S. PopA, On a class of $\mathrm{II}_{1}$ factors with at most one Cartan subalgebra. Ann. of Math. 172 (2010), 713-749.

[Pa77] W. L. PAschke, Integrable group actions on von Neumann algebras. Math. Scand. 40 (1977), 234-248.

[PiPo86] M. Pimsner, S. PopA, Entropy and index for subfactors. Ann. Sci. de l'É.N.S. (4) 19 (1986), 57-106.

[Pi95] G. PISIER, Projections from a von Neumann algebra onto a subalgebra. Bull. Soc. Math. France 123 (1995), 139-153.

[Po83] S. PopA, Maximal injective subalgebras in factors associated with free groups. Adv. in Math. 50 (1983), 27-48.

[Po86] S. PoPA, Correspondences. INCREST preprint (1986).

[Po01] S. PopA, On a class of type $\mathrm{II}_{1}$ factors with Betti numbers invariants. Ann. of Math. 163 (2006), 809-899.

[Po03] S. POPA, Strong rigidity of $\mathrm{II}_{1}$ factors arising from malleable actions of w-rigid groups I. Invent. Math. 165 (2006), 369-408.

[Sh98] D. Shlyakhtenko, Some applications of freeness with amalgamation. J. Reine Angew. Math. 500 (1998), 191-212.

[To18] R. Tomatsu, Centrally free actions of amenable $C^{*}$-tensor categories on von Neumann algebras. Preprint. arXiv:1812.04222.

[Va05] S. VAES, Strictly outer actions of groups and quantum groups. J. Reine Angew Math. 578 (2005), 147-184.

[Va09] S. VAES, An inner amenable group whose von Neumann algebra does not have property Gamma. Acta Math. 208 (2009), 389-394.

[Wo74] S. L. Woronowicz, Selfpolar forms and their applications to the $\mathrm{C}^{*}$-algebra theory. Rep. Math. Phys. 6 (1974), 487-495.

[Xu05] Q. Xu, A description of $\left(C_{p}\left[L_{p}(M)\right], R_{p}\left[L_{p}(M)\right]\right)_{\theta}$. Proc. Roy. Soc. Edinburgh Sect. A 135 (2005), 1073-1083.

Siena College Department of Mathematics, 515 Loudon Road, Loudonville, NY 12211, USA

E-mail address: jbannon@siena.edu

RIMS, KYOTO UNIVERSITY, 606-8502 JAPAN

E-mail address: amine.marrakchi@math.u-psud.fr

RIMS, KYoto UNIVERSITY, 606-8502 JAPAN

E-mail address: narutaka@kurims.kyoto-u.ac.jp 